\title{
Modélisation mathématique et simulation numérique d'écoulements à surface libre
}

\author{
Mathematical modelling and numerical simulation \\ of free surface flows
}

\author{
J.P. Vila \\ Centre de mathématiques appliquées (École Polytechnique) \\ et École nationale du génie rural, des eaux et des forêts
}

De nombreux phénomènes physiques intéressant le domaine de l'environnement sont concernés par les écoulements de fluides à surface libre; on s'intéresse ici essentiellement à la modélisation des phénomènes suivants :

- onde de crue due à la rupture d'un barrage;

- avalanche de neige dense;

- onde de submersion d'une retenue dûe à une avalanche ou à un glissement de terrain.

Sous certaines hypothèses ces trois phénomènes se modélisent à l'aide des équations de St-Venant. Dans les trois cas envisagés on est en présence d'écoulements transitoires avec de fortes discontinuités : le front de crue, le front d'avalanche ou la vague de submersion, suivant le cas. La présence de ces singularités provoque quelques difficultés lorsqu'on s'attaque à la simulation sur ordina- teur de ces phénomènes. De nombreux auteurs ont néanmoins abordé ce genre de simulation; les méthodes utilisées faisaient appel, pour la plupart, à un traitement séparé de ces discontinuités (méthode du "shock-fitting "). Les progrès récents, effectués dans l'analyse numérique des systèmes hyperboliques non linéaires, dont relèvent les équations de St-Venant, permettent d'envisager de nouvelles méthodes plus performantes et moins coûteuses.

Dans la première partie on expose le modèle mathématique et sa formulation conservatrice, dans la seconde partie on traite de l'approximation numérique des équations, dans la troisième partie on présente des tests de validation du modèle, ainsi que des applications aux problèmes déjà cités.

\section{Modélisation mathématique}

Sous les hypothèses suivantes :

(i) l'écoulement est monodimensionnel,

(ii) la distribution des pressions est hydrostatique,

(iii) les effets de viscosité à l'intérieur du fluide sont négligeables par rapport aux frottements externes, on écrira une équation de conservation de la masse, et une équation de conservation de la quantité de mouvement.

Avec :

$z=$ tirant d'eau

$S=$ section mouillée $(S(z))$

$L=$ largeur au miroir $\left(=S_{\text {.. }}\right)$

$Q=$ débit moyen dans une section

$i(x)=$ pente du canal

$F(Q, S, x)=$ frottement (visqueux, laminaire ou autre)

$g=$ accélération de la pesanteur

$x=$ abscisse $; t=$ temps

Figure 1 - Coupe d'une section.

LA HOUILLE BLANCHE/N $\mathrm{N}^{\circ}$ 6/7-1984 
on obtient classiquement :

$S_{1}+Q_{\mathrm{r}}=0$

$Q_{1}+\left(Q^{2} / S\right)_{x}+g S z_{x}=g S(i(x)-F(Q, S, x))$

système qui peut se mettre sous la forme suivante, dite, conservative :

$$
\begin{array}{lc} 
& U_{t}+f(U, x)_{-\mathrm{r}}=G(U, x) \\
\text { avec } & \begin{array}{l}
U=(S, Q) \\
\end{array} \\
& f(U, x)=\left(Q, Q^{2} / S+p(S, x)\right) \\
p(S, x)=g & \int_{0}^{z}(z-y) L(y) d y \\
\text { et } & G(U, x)=g S(i(x)-F(Q, S, x))
\end{array}
$$

Les hydrauliciens ont l'habitude de présenter séparément les équations aux discontinuités (i.e. le ressaut mobile) :

$\sigma$ indique la vitesse du ressaut et [ ] indique le saut d'une quantité de part et d'autre du ressaut.

$$
\begin{aligned}
& {[Q]=\sigma[S]} \\
& {\left[Q^{2} / S+p(S, x)\right]=\sigma[Q]}
\end{aligned}
$$

que l'on peut aussi écrire :

$$
[f(U)=\sigma[U]
$$

On démontre alors que toute solution faible (i.e. au sens des distributions) de (3), vérifie (6) (ceci résulte de la forme conservatrice du système); cette remarque est fondamentale pour la suite; il est en effet possible de concevoir des méthodes numériques qui respectent cette propriété et traitent les discontinuités comme des points ordinaires (donc avec une grande souplesse), c'est l'objet de la partie suivante.

$$
U_{i}^{n+1}=U_{i}^{n}-r\left(f_{j+1 / 2}^{n}-f_{i-1 / 2}^{n}\right)
$$

où le flux numérique $f_{j+\Gamma / 2}^{n}=h\left(U_{j}^{n}, U_{j+1}^{n}\right)$ vérifie :

$$
h(U, U)=f(U)
$$

Il est alors possible, en respectant cette règle, de construire des schémas traitant les discontinuités comme des points courants (schémas de Lax-Friedrichs [5], et de Godounov [4]); ces dernières méthodes ([5], [4]) relativement anciennes ont été assez peu utilisées, et cela malgré leur robustesse, elles ont en effet un défaut majeur : leur manque de précision.

Les progrès récents ont porté sur le développement de méthodes fiables, précises au second ordre. On peut utiliser à cet effet la technique d'antidiffusion-correction d'un schéma du premier ordre, c'est le point de vue que l'on adopte ici, en voici le principe :

On part d'un schéma du premier ordre, "robuste " (Godounov, par exemple)

$$
\bar{U}_{j}^{n+1}=U_{j}^{\prime \prime}-r\left(f_{j+1 / 2}^{n}-f_{j-1 / 2}^{n}\right)
$$

on calcule ensuite les coefficients d'antidiffusion $a_{j+1 / 2}^{n+1}$, qui permettent avec

$$
U_{j}^{n+1}=\bar{U}_{j}^{n+1}-\left(a_{j+1 / 2}^{n+1}-a_{j-1 / 2}^{n+1}\right)
$$

d'atteindre le second ordre en précision, on obtient ainsi un schéma du type Lax Wendroff, instable aux chocs; on est donc amené à corriger les coefficients " a ", la technique utilisée est inspirée de celle développée par Leroux [6] (voir aussi Vila [10]), après correction on calcule la solution définitive avec (9).

On n'a pas abordé ici les équations bidimensionnelles de St-Venant, elles ont aussi une forme conservatrice, analogue à (3), et on peut utiliser les méthodes précédentes :

Il suffit d'appliquer aux schémas monodimensionnels les techniques de direction alternées (approche différence-finies), ou les techniques volumes finis (si l'on veut avoir des géométries plus réalistes), pour obtenir des schémas bidimensionnels efficaces.

Les méthodes aux différences finies destinées à résoudre les problèmes d'hydraulique sont déjà anciennes (cf. [2], [8], [9]); elles font, pour la plupart, appel aux courbes caractéristiques. Ces schémas étant instables au voisinage des discontinuités de la solution, la technique du " shockfitting" a été introduite : l'équation (6) du ressaut est utilisée comme condition à la limite du problème, séparant ainsi la résolution dans les différentes zones régulières. Ces méthodes nécessitent l'utilisation de paramètres numériques (dépendants du problème, du maillage...) et sont particulièrement délicates à concevoir et à utiliser dès que l'on s'attaque à des situations complexes du type : réflexions d'ondes de choc, interactions de ces ondes entre elles, obstacles... etc.

On définit alors la consistance d'un schéma avec la forme conservatrice des équations :

- en utilisant les notations différences finies classiques : $D x$, le pas d'espace; $D t$, le pas de temps; $r=D t / D x$; et $U_{j}^{n+1}=U(j D x,(n+1) D t)$, un schéma est consistant avec la forme conservatrice (3.a) des équations s'il peut s'écrire :

\section{Approximation numérique}

\section{Validation et applications du modèle}

\section{Rupture de barrage et validation du modèle}

On ne connait, que dans de rares cas, des solutions analytiques aux équations de St-Venant instationnaires, un exemple particulièrement intéressant est celui de la rupture de barrage sur fond plat, dans un canal uniforme parfaitement lisse. Cet exemple fourni un test de validation du modèle; la figure 2 représente l'état initial, la solution exacte (fig. 3), est constituée d'une onde de détente et d'un ressaut mobile, séparés par un état constant; la figure 4 compare à l'instant $t=48 \mathrm{~s}$. la solution exacte à la solution calculée, elle montre bien les performances de la méthode. Un code de calcul permettant le calcul d'ondes de rupture réelles, utilisant ces principes est en cours de développement au CEMAGREF, sa simplicité 


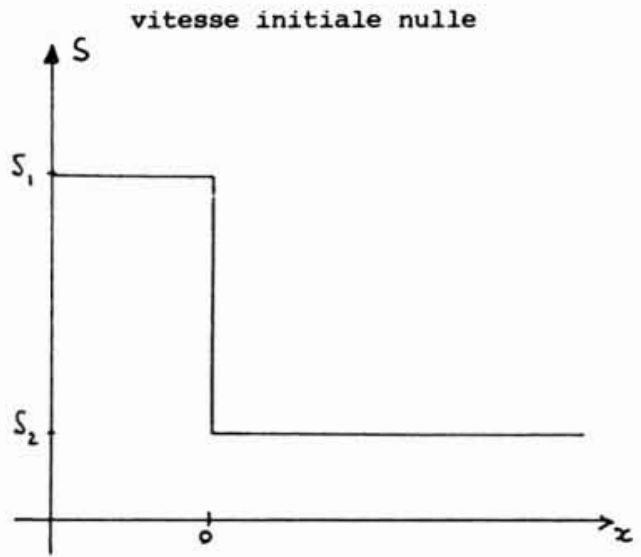

Figure 2 - Etat initial.

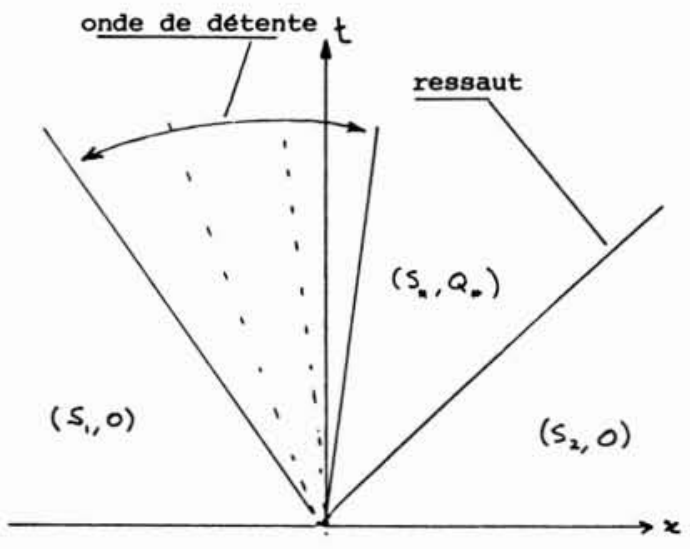

Figure 3 - Solution exacte.

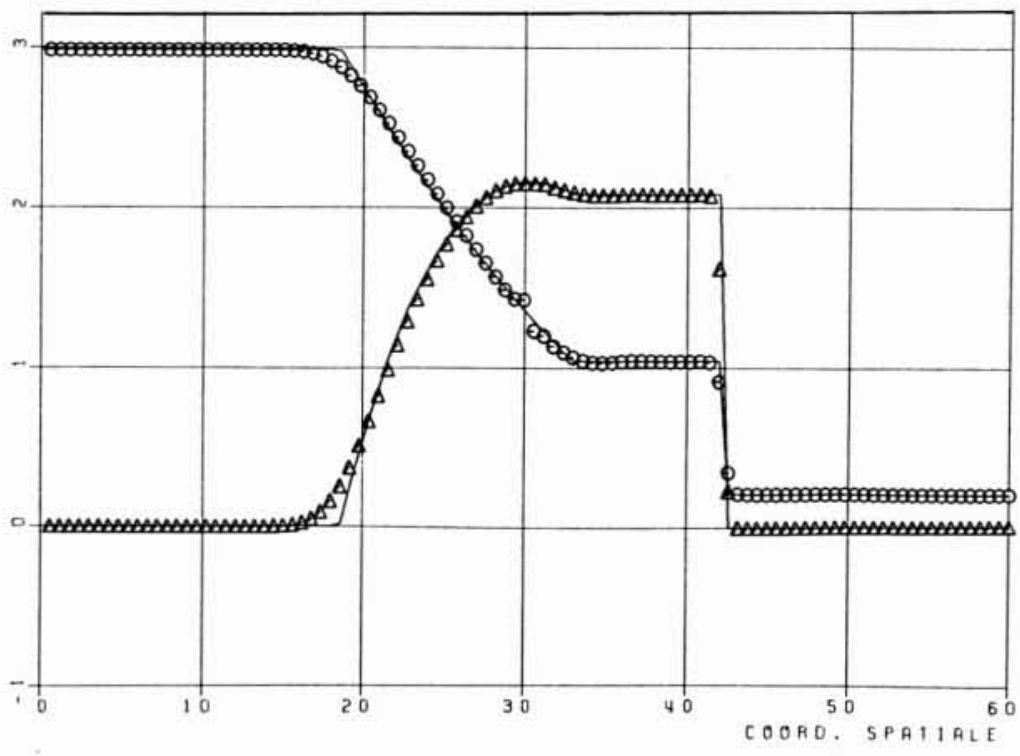

Figure 4-Comparaison solution exacte - solution calculée. Maillage : $100 \mathrm{pts} \quad \mathrm{O}$ : surf. mouill. (calculée) $\Delta$ : débit (calculé)

- : solution exacte

(schéma explicite), sa précision et sa robustesse doivent permettre un net progrès dans le calcul des ruptures fortes.

\section{Avalanches de neige dense}

On peut aborder, maintenant, un domaine moins traditionnel, d'application des équations de St-Venant: la modélisation des avalanches de neige dense.

Si l'on pose :

$$
\begin{gathered}
S^{*}=\rho S \\
Q^{*}=\rho Q \\
p^{*}(\rho, S, x)=\rho p(S, x)
\end{gathered}
$$

les équations (1)-(2) sont encore vérifiées, avec $S^{*}, Q^{*}, p^{*}$ à la place de $S, Q$ et $p$; il est alors nécessaire de se donner une loi phénoménologique, décrivant la densité : $\rho=\rho\left(S^{*}, Q^{*}, x\right)$, pour fermer le problème.

Brugnot et Pochat ont montré dans [1], les possibilités d'un tel modèle; la méthode exposée précédemment, appliquée à ce problème a pu être programmée sur micro-ordinateur (explicite, elle ne nécessite pas l'inversion de gros systèmes linéaires), elle permet avec des temps de calcul inférieurs à $15 \mathrm{mn}$ (très acceptables sur un micro) de simuler une avalanche. Les études précédentes ont montré le sensibilité du modèle aux différents paramètres : coefficients de frottement, conditions initiales, hauteur de reprise.

Une meilleure connaissance du phénomène " avalanche " nécessite une confrontation des résultats expérimen- 
taux avec ce genre de simulation. Le CEMAGREF a réalisé des déclenchements artificiels (cf.[3]) au cours desquels, de nombreuses mesures de vitesse ont été enregistrées par stéréophotogrammétrie. La technique suivante permet de mieux cerner le problème de la hauteur de reprise :

On dispose en effet, par stéréophoto, de mesures de $d h$ (la hauteur du front) et de $\sigma$ (la vitesse du front); en confrontant ces données avec (4) et (5), (les équations du ressaut), on détermine $h O$, la hauteur de reprise; hauteur que l'on peut alors comparer à des mesures, ou utiliser dans l'initialisation du modèle numérique, permettant ainsi une meilleure identification des autres paramètres (termes de frottement, en particulier); la précision actuelle des mesures de stéréophoto sur les sites du CEMAGREF ne permet pas encore une utilisation satisfaisante de cette technique.

Cependant, l'utilisation de l'outil numérique permet ici de mieux analyser les phénomènes physiques et cela malgré la simplicité du modèle vis à vis de la complexité de l'écoulement réel.

\section{Onde de submersion}

On présente, pour terminer une application du modèle, à un problème qui peut intéresser les retenues en haute altitude :

Une avalanche termine sa course dans une retenue; il s'agit d'évaluer les surcotes provoquées par cet accident. Noda [7] a étudié ce problème à l'aide d'une théorie simplifiée. On envisage ici une théorie plus fine, permettant de mener à bien des calculs dans les cas réels, les équations de St-Venant modélisent la aussi le phénomène, le mouvement de l'avalanche sera considéré comme une donnée externe (ce mouvement pourrait être évalué à l'aide de la méthode étudiée au $\S$ b).

Cette avalanche va intervenir de deux façons: d'une part elle modifie la topographie de la retenue, en occupant un volume initialement occupé par de l'eau, d'autre part elle entraîne l'eau dans son mouvement, par le biais du frottement à l'interface " avalanche-eau ".

Le travail effectué a consisté, pour l'instant, à tester le modèle sur des cas monodimensionnels, afin de résoudre les problèmes pouvant se poser aux faibles profondeurs avec les seconds membres. La division nivologie du CEMAGREF entreprend actuellement la modélisation physique d'un tel écoulement, et compte coupler cette étude à une simulation numériqué.

Les figures $6,7,8,9$, représentent à des instants successifs les résultats de calculs, hauteur (courbe supérieure) et vitesse (courbe inférieure), simulant l'onde de submersion provoquée dans une retenue rectangulaire par une avalanche (elle est représentée par le coin en bas à gauche des figures, mais elle est déjà arrêtée aux instants représentés).

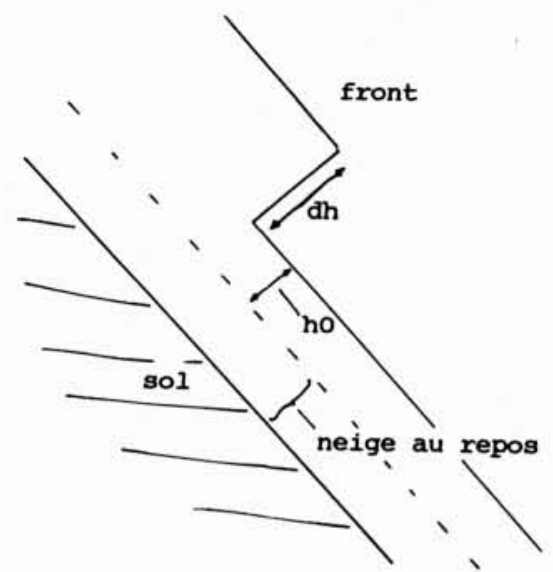

Figure 5 - Hauteur de reprise.
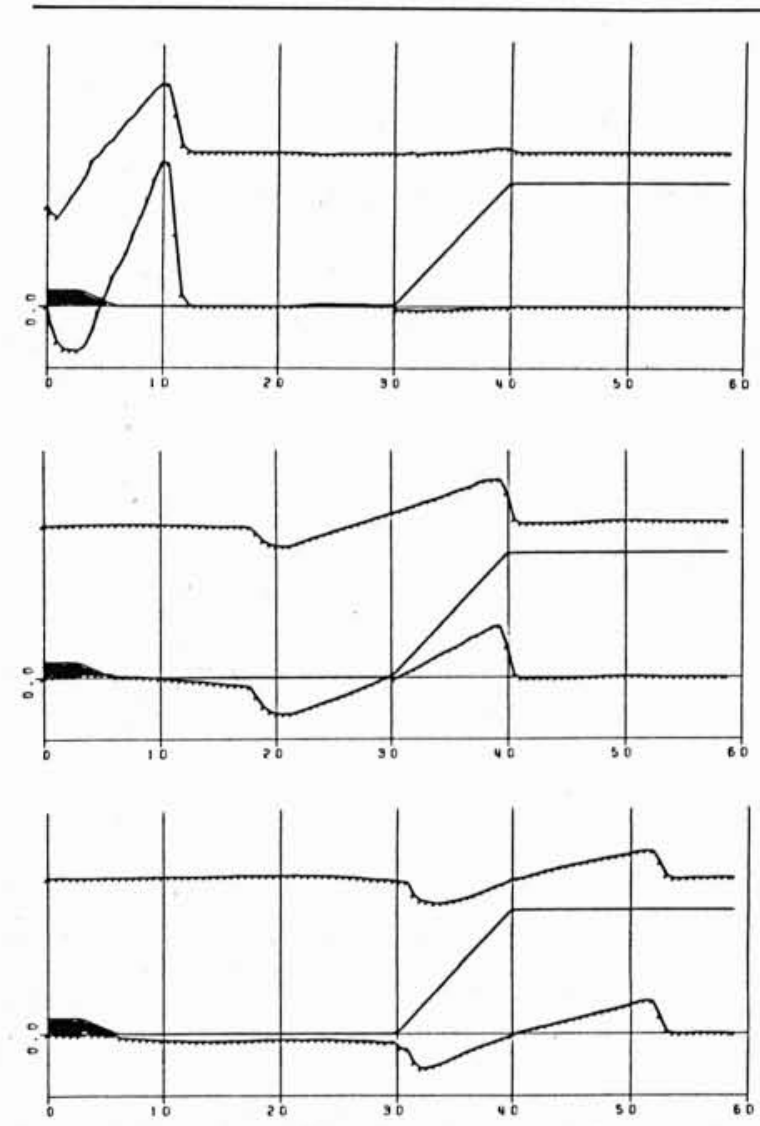

8

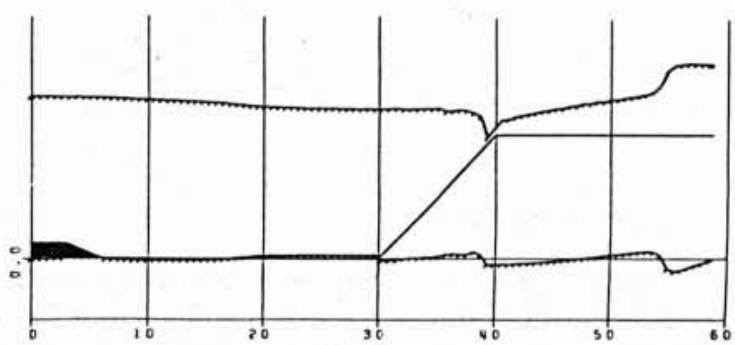

9

Figure $6-\mathrm{T}=5.06 \mathrm{~s}$, on peut noter la forte courbure de l'onde de détente amont.

Figure $7-T=20.3 \mathrm{~s}$, le front est encore raide.

Figure $8-\mathrm{T}=30.1 \mathrm{~s}$, la vague se ralentit sur la plage.

Figure $9-\mathrm{T}=40.3 \mathrm{~s}$, réflexion du front sur la limite droite, inversion des vitesses. 


\section{Conclusion et commentaires}

On a présenté ici trois types de simulations numériques d'écoulements à l'aide d'un modèle St-Venant; dans le premier et le troisième cas les hypothèses sont telles que l'on peut envisager une utilisation effective de la méthode à l'évaluation des risques dus à une rupture de barrage ou à une onde de submersion (le recours à une simulation physique couplée n'étant pas nécessairement exclu - cf. $\S \mathrm{c}-$ ). Dans le cas des avalanches de neige dense il faut rester moins ambitieux et considérer l'outil numérique comme un moyen de tester certaines hypothèses physiques; on est encore trop loin de la réalité de l'écoulement surtout dans la zone d'arrêt de l'avalanche pour se permettre d'être plus affirmatif, tout au plus, peut-on envisager l'utilisation de codes bidimensionnels pour évaluer l'influence d'un ouvrage de protection placé sur la trajectoire d'une avalanche.

\section{Références bibliographiques}

[1] BRUGNOT G., POCHAT R. - Numérical simulation study of avalanches. Journal of Glaciology, 27, 1981, 95, p. 77-88.

[2] COURANT R., HILBERT D. - Methods of mathematical physics, vol. II. Interscience, N.Y., 1962.

[3] C.T.G.R.E.F. div, nivologie, - La stéréophotogrammétrie à cadence rapide d'avalanches. Informations techniques du CTGREF, 1980 , cahier 39 , fasc. 8 .

[4] GODOUNOV S.K. - Méthodes aux différences finies. Mat. Sbornik, 47, 1959, 271-306.

[5] LAX P.D. - Hyperbolic systems of conservations laws II. Comm. Pure Appl. Math., 10, 1957, 537-566.
[6] LEROUX A.Y. - Thèse d'état. Université de Rennes, 1979.

[7] NODA E. - Water waves generated by landslides. Proc., Am. Soc. Civ. Eng., J. Waterw. Harbors Div. 96 (WW4), 1970, 835-855.

[8] RICHTMEYER R.D., MORTON K.W. - Difference methods for initial value problems. Interscience N.Y., 1967.

[9] STOKER J.J. - Water waves. Interscience N.Y., 1957.

[10] VILA J.P. - Simplified Godounov schemes for $2 \times 2$ systems of conservation laws. Rapport interne (à paraître), C.M.A.P., Ecole Polytechnique.

\section{Discussion}

M. le Président remercie M. VILA et souligne l'importance de ces phénomènes pour les lacs de la Cordillère Blanche au Pérou.

M. LEVY demande le point exact de la différence entre cette méthode et celle de Pochat.

M. VILA répond que pour cet auteur il y avait séparation au niveau numérique entre le choc et la propagation, par contre ici c'est le résultat du calcul qui donne le choc.

$M$. le Président s'étonne du caractère confidentiel des références citées pour ces méthodes d'autant plus qu'elles peuvent s'appliquer à des problèmes très divers.
M. VILA répond que c'est dû à leur développement récent, essentiellement en hydrodynamique et que malgré leur complexité elles suivent de très près les problèmes physiques. Elles s'appliquent particulièrement aux phénomènes transitoires mais pour des phénomènes plus rapides sans discontinuité, il existe des méthodes plus adaptées et moins coûteuses.

M. le Président remercie M. VILA de son exposé et clôt la séance qu'il juge très intéressante en se félicitant que ces communications soient publiées. 


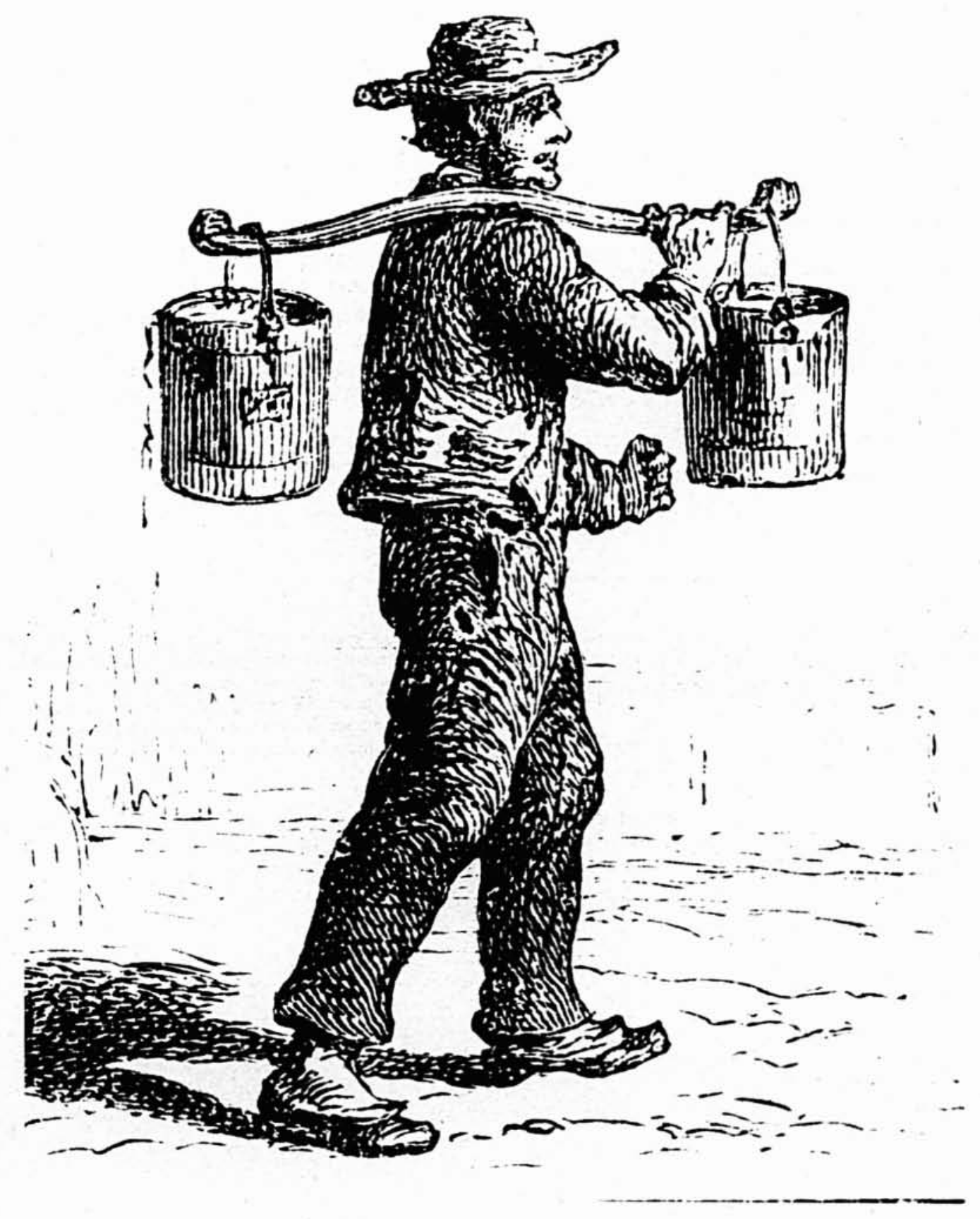

